'Escuela de Kinesiología, Universidad Católica Silva Henríquez. Santiago, Chile. aKinesiólogo, Magister en Educación.

bKinesiólogo, Licenciado en Kinesiología.

Fuente de apoyo financiero: Dirección de Investigación y Postgrado, Universidad Católica Silva Henríquez.

Los autores declaran no tener conflictos de interés.

Recibido el 19 de agosto de 2020, aceptado el 25 de mayo de 2021.

Correspondencia a: Jorge Ugarte LI. Escuela de Kinesiología, Universidad Católica Silva Henríquez. Santiago de Chile. jugarte@ucsh.cl

\section{Sensibilidad y especificidad de la prueba Timed Up and Go. Tiempos de corte y edad en adultos mayores}

\author{
JORGE UGARTE LL. ${ }^{1, a}$, FELIPE VARGAS R., ${ }^{1, b}$
}

\section{Timed up and go values in older people with and without a history of falls}

Background: Timed Up and Go (TUG) is used to assess the risk of falling of older people. Aim: To evaluate the sensitivity and specificity of TUG as a predictor of falls in older adults. Material and methods: TUG was measured in 148 independent community-dwelling older adults aged $75 \pm 7$ years (85\% women). Of these, 58 reported having a fall in the previous year. Analysis of the ROC (Receiver Operating Characteristic) curve was performed to assess the sensitivity and specificity of common cut-off times used in clinical practice. The times required to perform the TUG as quickly as possible (best fitted time) and at the usual pace (common time) were registered. Results: Participants with a history of falls had higher TUG times than their counterparts who did not fall (10.9 \pm 3.9 and $9.2 \pm 2.6$ s, respectively). By age groups, only in the 60-69 age group the differences between those who fell and those who did not, were statistically significant $(p<0.05)$. A cut-off of 9 s generates the better sensitivity and specificity for the test (0.60 and 0.57, respectively). For 60-69 age group the best cut-off time is 8.2s, with an increase in sensitivity and specificity to 0.73 and 0.68 , respectively. Conclusions: There were differences in TUG values between participants with and without a history of falls. Determining TUG cut-offvalues by age groups improves the sensitivity and specificity of the test, especially in the 60-69 age range.

(Rev Med Chile 2021; 149: 1302-1310)

Key words: Aging; Physical Functional Performance; ROC Curve.
$\mathrm{L}$ a prueba "Up and Go" fue originalmente diseñada en 1985 como una herramienta para evaluar balance ${ }^{1}$. En 1991 se introdujo la versión cronometrada para evaluar movilidad de los adultos mayores (AM), y desde entonces, el Timed Up and Go (TUG, por sus siglas en inglés) ha sido ampliamente usado para evaluar a AM y también como predictor de caídas ${ }^{2}$. La prueba requiere que el participante se incorpore desde una silla, camine tres metros rodeando un obstáculo, camine de regreso y se siente nuevamente, retomando su posición original. El TUG tiene una moderada correlación con el riesgo de caídas ${ }^{3}$ y ha demostrado una muy buena confiabilidad test-retest (ICC 0,80-0,99), no obstante, su validez y sensibilidad varían entre poblaciones. Existen cambios mínimos detectables entre poblaciones (incluyendo poblaciones con diferentes morbilidades) con tiempos de 1,6 a 4,5 segundos ${ }^{4,5}$. Se ha propuesto que un tiempo de rendimiento en el TUG sobre 13,5 segundos, es predictor para riesgo de caídas en AM que viven en comunidad, sin embargo, este nivel de predicción varía de acuerdo a la condición física de los AM (siendo más útil en AM con bajas capacidades funcionales) $)^{6,7}$. Un metaanálisis concluyó que los tiempos del TUG 
para el rango 60-99 años, tenía un promedio de 9,4 segundos con un intervalo de confianza de 8,9 - 9,9 segundos a 95\%. Lo anterior muestra una alta homogeneidad; los resultados son diferentes entre rangos etarios, y valores por encima del intervalo de confianza muestran lógicamente un peor rendimiento, no obstante, dicho rendimiento no se comparó frente al riesgo de caídas o una historia previa de estas ${ }^{8}$.

Asai y cols. ${ }^{9}$ concluyeron que la aplicación del TUG debe ser complementada con tareas motoras o cognitivas para aumentar la predicción de caídas en individuos con capacidades funcionales conservadas. Kojima, Masud y Kendrick ${ }^{10}$ afirman que el TUG tiene un valor predictivo aceptable si las co-morbilidades como medicación o historial de caídas se consideran, estableciendo 12,6 segundos como tiempo para afirmar que existe un alto riesgo de caída. Otros estudios recomiendan no aplicar el TUG aisladamente en la evaluación del AM debido a su limitada capacidad para predecir caídas. Esto se debe a que se desconoce como la prueba puede predecir caídas y que condiciones lo determinan, no obstante, el TUG es una herramienta masiva y fácil de aplicar (debido principalmente a sus ventajas operativas, y porque es altamente reproducible $)^{11,12}$.

Existen varios factores que contribuyen a las caídas en AM, por ejemplo, alteraciones en el balance postural o disminución de la fuerza muscular en extremidades inferiores ${ }^{13}$. Las alteraciones de la marcha son también un factor importante en la evaluación del riesgo de caídas, sin embargo, estas por sí solas, son insuficientes para evaluar tareas motoras más complejas ya que se omiten otras variables importantes propias del envejecimiento. El TUG evalúa balance, fuerza de extremidades inferiores y equilibrio, pero no evalúa conductas motoras más elaboradas, las cuales reflejan más fielmente actividades del diario vivir en las cuales ocurren los accidentes, como, por ejemplo, el aseo personal'.

También la sensibilidad (Sn), especificidad (Sp) y los tiempos de corte del TUG para predecir el riesgo de caídas, varían entre poblaciones, rangos etarios, entre los cuales existen distintos tiempos de rendimiento ${ }^{3,6,9,11,14}$.

El objetivo de esta investigación entonces fue analizar la Sn y Sp del TUG entre AM que han sufrido caídas dentro de un año y aquellos que no, así como los tiempos de corte según grupo etario.

\section{Métodos}

\section{Población y diseño}

El diseño de este estudio es de cohorte, retrospectivo, que fue realizado en centros de actividades para AM en Santiago de Chile entre 2018 y 2019 y que fue autorizado por el Servicio Nacional del Adulto Mayor (SENAMA) e institucionalmente por la Universidad Católica Silva Henríquez. Los AM que se atendieron en estos centros eran independientes y poseían plenas facultades mentales para responder preguntas $y$ seguir instrucciones. Antes de participar como voluntarios, se les explicó el propósito de la investigación y firmaron un consentimiento informado. Novecientos veinte y siete usuarios estaban distribuidos en los 12 centros (Media $77 \pm 13$ ). Seis centros fueron escogidos randomizadamente, por lo que se realizaron 149 evaluaciones. Los criterios de inclusión fueron: AM sobre 60 años, capaces de seguir instrucciones, sin lesiones musculoesqueléticas recientes que afecten la marcha. Un individuo fue excluido a causa del uso de silla de ruedas. El uso de ayudas técnicas se permitió en 13 casos $(8,4 \%)$. Ningún evento adverso ocurrió durante las evaluaciones. De esta forma, se evaluaron 148 AM, de los cuales 141 casos se incluyeron en el análisis. La muestra entonces, se componía de 120 mujeres (85\%) y 21 hombres (17\%) (Tabla 1).

\section{Procedimiento}

En primer lugar, se realizaron mediciones antropométricas como la estatura, medida con un estadiómetro (ADE ${ }^{\circledR}$ MZ10042 Alemania) y el peso, evaluado con una balanza digital (SECA Sensa 804) (Tabla 2). Posteriormente, un investigador aplicó el Mini-Mental Test y solicitó información sobre caídas dentro de los últimos 12 meses, considerando la definición de "caídas accidentales" aceptada por el Medical Subject Heading (MeSH), las cuales ocurren al deambular o tropezar y pueden resultar en alguna lesión. Para esta investigación, un "caedor" se consideró como aquel AM que registró un accidente dentro del último año. Importante mencionar que los AM que sufrieron caídas y que resultaron en lesiones graves fueron excluidos de la muestra. Finalmente, culminado el proceso de recolección de información, otro investigador aplicaba el TUG en los AM seleccionados. 
Tabla 1. Grupos etarios y distribución según género

\begin{tabular}{|c|c|c|c|c|c|}
\hline & & & \multicolumn{2}{|c|}{ Género } & \multirow[t]{2}{*}{ Total } \\
\hline & & & Hombre & Mujer & \\
\hline \multirow[t]{8}{*}{ Grupo etario } & $60-69$ & Recuento & 5 & 32 & 37 \\
\hline & & \% dentro de Grupo Etario & $13,5 \%$ & $86,5 \%$ & $100,0 \%$ \\
\hline & $70-79$ & Recuento & 7 & 57 & 64 \\
\hline & & \% dentro de Grupo Etario & $10,9 \%$ & $89,1 \%$ & $100,0 \%$ \\
\hline & $80-89$ & Recuento & 9 & 29 & 38 \\
\hline & & $\%$ dentro de Grupo Etario & $23,7 \%$ & $76,3 \%$ & $100,0 \%$ \\
\hline & $90+$ & Recuento & 0 & 2 & 2 \\
\hline & & \% dentro de Grupo Etario & $0,0 \%$ & $100,0 \%$ & $100,0 \%$ \\
\hline \multirow[t]{2}{*}{ Total } & & Recuento & 21 & 120 & 141 \\
\hline & & \% dentro de Grupo Etario & $14,9 \%$ & $85,1 \%$ & $100,0 \%$ \\
\hline
\end{tabular}

Todas las entrevistas y evaluación del TUG fueron realizadas por distintos kinesiólogos, de tal forma que quienes evaluaron el TUG eran ciegos al historial de caídas de los AM.

\section{Timed Up and Go}

Se indicó a los participantes incorporarse desde una silla, sin usar brazos, y caminar alrededor de un cono ubicado a tres metros de esta, para luego regresar y sentarse. Los participantes sabían que la prueba era cronometrada, por ende, se les indicó que lo hicieran "como habitualmente camina", evitándose la instrucción "lo más rápido posible". Un evaluador explicó y demostró la prueba a los participantes. Se registraron tres mediciones consecutivas, considerándose el mejor tiempo de los tres intentos para su análisis. Los resultados fueron registrados con un decimal. Se utilizó la clasificación del Examen Preventivo del Adulto Mayor (EMPAM) como referencia para complementar la información obtenida tanto en las entrevistas como en las mediciones y su posterior análisis (Normal $\leq 10$ segundos; Riesgo leve de caída 11 a 20 segundos; Alto riesgo de caída $>20$ segundos).

\section{Análisis estadístico}

Para análisis de los datos se utilizó el software Stata $^{\circledR} / \mathrm{MP} 13$.

Se compararon las medias entre caedores y no caedores usando prueba-t para muestras independientes.

El análisis de la curva ROC (Receiver Operating Characteristic) se usó para determinar la
Sn y Sp del TUG en diferentes tiempos de corte (95\% IC). Se usaron tiempos de 9, 10, 12 y 15 segundos respectivamente, para comparar la $\mathrm{Sn}$, Sp, e índices de probabilidad positivos y negativos entre otros.

Se utilizó la prueba de Chi cuadrado $\left(\chi^{2}\right)$ para evaluar la dependencia entre grupos para lo cual, la muestra fue segmentada en grupos etarios (6069, 70-79, 80-89, 90-99 años).

\section{Resultados}

\section{TUG}

Las medias son presentadas en Tabla 2, y las pruebas-t en Tabla 3. El análisis para la muestra total, refleja que $83 \mathrm{AM}$ se clasificaron como no caedores $(58,9 \%)$ y 58 AM se clasificaron como caedores $(41,1 \%)$.

Para la muestra completa, las medias (Caedores 10,9 segundos/No Caedores 9,2 segundos) son estadísticamente diferentes $(\mathrm{p}<0,05)$. Cuando las diferencias son re-testeadas por grupo etario, se observa que solo para el grupo 60-69 años, las medias son estadísticamente diferentes (Caedores 9,7 segundos/No Caedores 7,6 segundos). Valores atípicos en el TUG se ubicaron sobre 21,5 segundos, incluyendo a 5 caedores y 2 no caedores (Figura 1).

Un hallazgo esperable, es que los tiempos promedio y la desviación estándar (como medida de variabilidad) incrementa con la edad, y siempre es mayor en el grupo de caedores. 
Sensibilidad y especificidad de prueba Timed Up and Go - J. Ugarte et al

Tabla 2. Estadística descriptiva de la muestra

\begin{tabular}{|c|c|c|c|c|c|c|c|c|c|c|}
\hline \multirow{2}{*}{\multicolumn{2}{|c|}{ Rango edad }} & \multicolumn{3}{|c|}{ Ambos grupos } & \multicolumn{3}{|c|}{ No caedores } & \multicolumn{3}{|c|}{ Caedores } \\
\hline & & Edad & TUG & IMC & Edad & TUG & IMC & Edad & TUG & IMC \\
\hline \multirow[t]{4}{*}{$60-69$} & $n$ & 37 & 37 & 36 & 22 & 22 & 22 & 15 & 15 & 14 \\
\hline & media & 65,9 & 8,5 & 27,9 & 66,3 & 7,6 & 26,7 & 65,4 & 9,8 & 29,8 \\
\hline & SD & 2,7 & 2,4 & 6,3 & 2,8 & 1,7 & 5,9 & 2,6 & 2,7 & 6,7 \\
\hline & p50 & 66 & 8,1 & 26,2 & 66,5 & 7,5 & 25,5 & 66 & 10 & 29,1 \\
\hline \multirow[t]{4}{*}{$70-79$} & $n$ & 64 & 64 & 64 & 41 & 41 & 41 & 23 & 23 & 23 \\
\hline & media & 74,4 & 10 & 30,2 & 74,2 & 9,4 & 31,5 & 74,7 & 11,1 & 27,9 \\
\hline & SD & 2,5 & 3,1 & 17,9 & 2,7 & 2,6 & 22,2 & 2,2 & 3,8 & 4 \\
\hline & p50 & 74 & 8,9 & 27,2 & 74 & 8,7 & 27,4 & 75 & 10 & 27 \\
\hline \multirow[t]{4}{*}{$80-89$} & $\mathrm{n}$ & 38 & 38 & 38 & 19 & 19 & 19 & 19 & 19 & 19 \\
\hline & media & 83,4 & 10,9 & 27 & 84,3 & 10,6 & 26,7 & 82,6 & 11,1 & 27,3 \\
\hline & SD & 2,3 & 3,6 & 3,9 & 2,6 & 2,9 & 3,8 & 1,6 & 4,2 & 4,1 \\
\hline & p50 & 83 & 10 & 26,4 & 84 & 10,3 & 27 & 82 & 10 & 26,1 \\
\hline \multirow[t]{4}{*}{$90+$} & $n$ & 2 & 2 & 2 & 1 & 1 & 1 & 1 & 1 & 1 \\
\hline & media & 93,5 & 15,7 & 25 & 93 & 10,5 & 23,7 & 94 & 20,8 & 26,4 \\
\hline & SD & 0,7 & 7,3 & 1,9 & - & - & - & - & - & - \\
\hline & p50 & 93,5 & 15,7 & 25 & 93 & 10,5 & 23,7 & 94 & 20,8 & 26,4 \\
\hline \multirow[t]{4}{*}{ Total } & $n$ & 141 & 141 & 140 & 83 & 83 & 83 & 58 & 58 & 57 \\
\hline & media & 74,9 & 9,9 & 28,7 & 74,7 & 9,2 & 29,1 & 75,2 & 10,9 & 28,1 \\
\hline & SD & 7,2 & 3,3 & 12,7 & 7,2 & 2,6 & 16,1 & 7,4 & 3,9 & 4,8 \\
\hline & p50 & 75 & 9,2 & 27 & 74 & 8,7 & 27 & 75 & 10 & 27 \\
\hline
\end{tabular}

$\mathrm{n}=$ casos, p50= percentil 50, SD=Desviación estándar. Edad en años, TUG en segundos, índice de masa corporal en kg/(m²).

Tabla 3. Resultados de comparación de medias entre grupos

\begin{tabular}{|c|c|c|c|c|c|c|c|c|c|}
\hline \multicolumn{6}{|c|}{ Estadísticas grupales } & \multicolumn{4}{|c|}{$\begin{array}{l}\text { TUG t-test para igualdad de medias } \\
\text { por historial de caídas }\end{array}$} \\
\hline \multicolumn{2}{|c|}{ Por grupo etario } & \multirow{2}{*}{$\begin{array}{l}\mathbf{n} \\
83\end{array}$} & \multirow{2}{*}{$\begin{array}{c}\text { media } \\
9,20\end{array}$} & \multirow{2}{*}{$\begin{array}{l}\text { SD } \\
2,63\end{array}$} & \multirow{2}{*}{$\begin{array}{l}\text { EEM } \\
0,29\end{array}$} & \multirow{2}{*}{$\begin{array}{c}\mathbf{t} \\
\text { (2-tailed) } \\
-2,988\end{array}$} & \multirow{2}{*}{$\begin{array}{c}\text { Sig. } \\
0,004 * *\end{array}$} & \multirow{2}{*}{$\begin{array}{c}\text { Diferencias } \\
\text { de las medias } \\
-1,73957\end{array}$} & \multirow{2}{*}{$\begin{array}{c}\text { Diferencias } \\
\text { EEM } \\
0,58222\end{array}$} \\
\hline Todos & No caedor & & & & & & & & \\
\hline & Caedor & 58 & 10,94 & 3,85 & 0,51 & & & & \\
\hline \multirow[t]{2}{*}{$60-69$} & No caedor & 22 & 7,62 & 1,67 & 0,36 & $-3,061$ & $0,004^{* *}$ & $-2,17912$ & 0,71185 \\
\hline & Caedor & 15 & 9,80 & 2,67 & 0,69 & & & & \\
\hline \multirow[t]{2}{*}{ 70-79 } & No caedor & 41 & 9,36 & 2,55 & 0,40 & $-1,975$ & 0,057 & $-1,74457$ & 0,8834 \\
\hline & Caedor & 23 & 11,11 & 3,78 & 0,79 & & & & \\
\hline \multirow[t]{2}{*}{$80-89$} & No caedor & 19 & 10,63 & 2,90 & 0,67 & $-0,431$ & 0,669 & $-0,50158$ & 1,16502 \\
\hline & Caedor & 19 & 11,13 & 4,17 & 0,96 & & & & \\
\hline \multirow[t]{2}{*}{$90-99$} & No caedor & 1 & 10,50 & & & & & & \\
\hline & Caedor & 1 & 20,83 & & & & & & \\
\hline
\end{tabular}

Casos (n), desviación estándar (SD), y error estándar de la media (EEM), Diferencias estadísticamente significativas se marcan con doble asterisco $\left.{ }^{* *}\right)$. Para toda la muestra las medias son estadísticamente diferentes en 1,7 segundos $(p<0,05)$. 


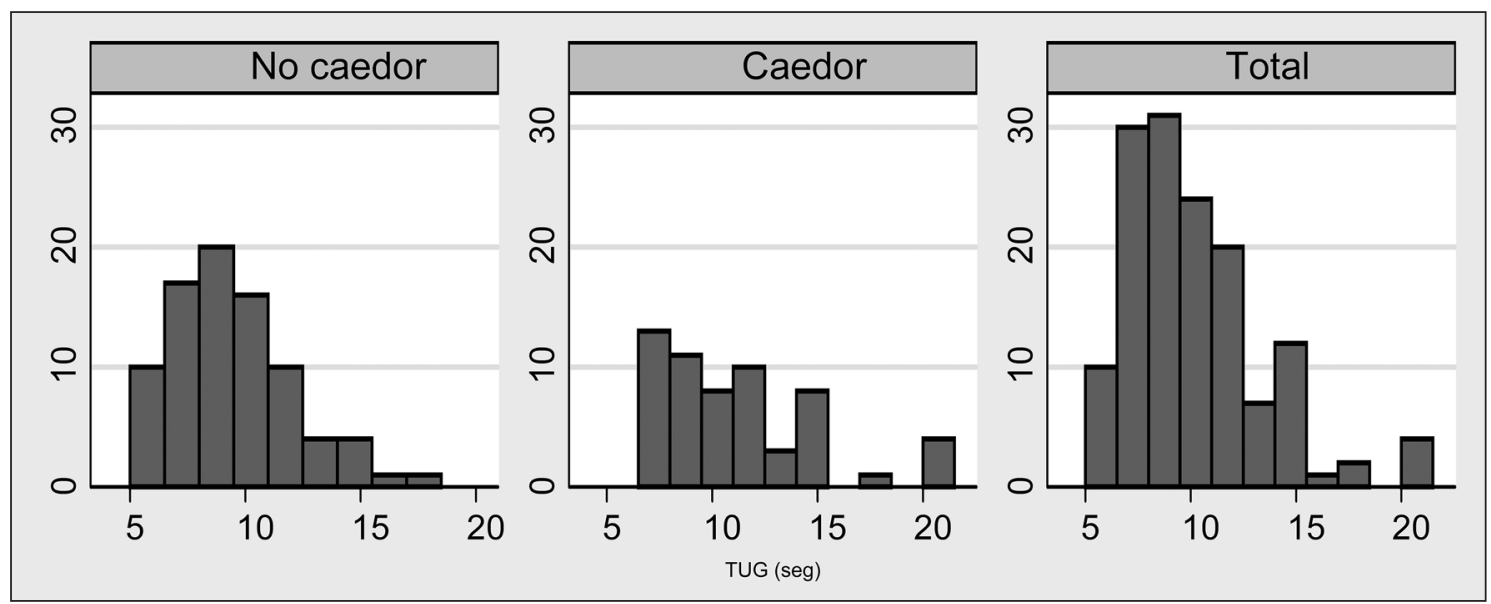

Figura 1. Frecuencias de distribución por grupo. Distribución de tiempo en el TUG. No caedores $(n=83)$, caedores $(n=58)$ y toda la muestra $(n=141)$.

\section{Género}

Se evaluó la asociación entre género e historia de caídas (caedor o no caedor) $\left(\chi^{2}=0,09\right.$; $\mathrm{p}=0,882)$. Se obtuvo el coeficiente de contingencia $(0,029 ; \mathrm{p}=0,758)$ y los Odds Ratio (OR) para Caedores Hombre/Mujer (OR Caedor Hombre/ Mujer $=0,86)$. El riesgo de caída para hombres es 0,38 y 0,48 para mujeres, con un Risk Ratio (RR) Hombre/Mujer de 0,91. Aunque la muestra estaba constituida principalmente por mujeres, esto indica independencia entre el género y caídas en este caso.

\section{Clasificación del TUG por grupos}

Se evaluó la clasificación de riesgo utilizada en el EMPAM y la historia de caídas. El valor de la prueba Chi-cuadrado $\left(\chi^{2}=7,85 \mathrm{p}=0,02\right)$ refleja que existe una asociación estadísticamente significativa entre estas variables.

\section{Análisis curva ROC}

La curva ROC que analizó los tiempos del TUG del grupo de AM caedores muestra un área $=0,63$, SD 0,046, $p=0,009$ (Figura 2, Tabla 4). La Sn y Sp para los diferentes tiempos son presentadas en la Tabla 4. El análisis de la curva sugiere que un tiempo de corte de 9,0 segundos, refleja la mejor $\mathrm{Sn}$ $(0,62)$ y $\mathrm{Sp}(0,54)$. Se observa entonces un grupo de riesgo con tiempos de corte de 9 segundos (tiempos menores a 9 segundos reflejarían bajo riesgo de caídas); la prueba Chi-cuadrado se repitió arrojando un valor $\chi^{2}=393 \mathrm{p}=0,047$ (Figura 3), lo cual refleja una mejor asociación entre rendimiento en el TUG e historia de caídas, mostrando que, para este tiempo de corte, la prueba puede diferenciar significativamente a los no caedores.

\section{Análisis por grupo etario}

La muestra se dividió en decenios que conformaron 4 grupos (60-69, 70-79, 80-89 y 90-99 años respectivamente) para su análisis (Figura 4).

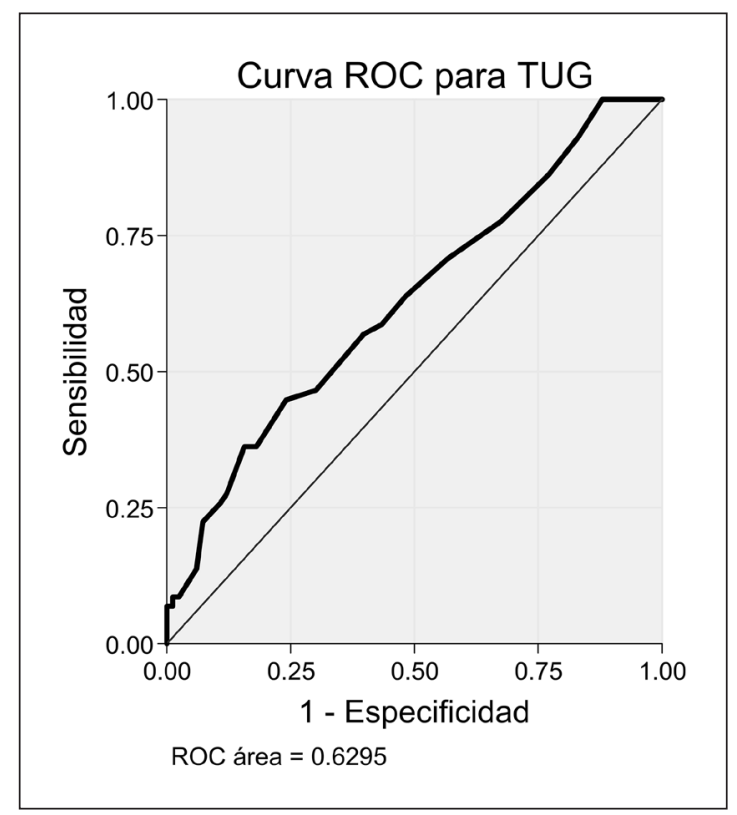

Figura 2. Curva ROC para la muestra. 
Tabla 4. Análisis detallado de las curvas ROC, TUG, Sn y Sp para los tiempos de corte usados comúnmente en guías clínicas en atención en salud, en relación a las historias de caídas en el último año

\begin{tabular}{|c|c|c|c|c|c|c|c|c|}
\hline Corte & $S n^{(a)}$ & $S p^{(b)}$ & $\mathbf{L R}+\mathbf{t}^{(\mathbf{c})}$ & $\mathbf{L R}_{-}^{-(d)}$ & + РoTO(e) & -PoTO(f) & +PoTP(g) & -PoTP(h) \\
\hline \multicolumn{9}{|c|}{ Total muestra $(n=141)$} \\
\hline \multicolumn{9}{|c|}{ ROC (¥) área 0,6296 (0,53558-0,72366) } \\
\hline 8,2 & 0,71 & 0,42 & 1,22 & 0,70 & 0,85 & 0,48 & 0,46 & 0,33 \\
\hline 9 & 0,62 & 0,54 & 1,36 & 0,70 & 0,95 & 0,49 & 0,49 & 0,33 \\
\hline 10 & 0,57 & 0,63 & 1,52 & 0,69 & 1,06 & 0,48 & 0,52 & 0,32 \\
\hline 12 & 0,36 & 0,84 & 2,31 & 0,76 & 1,61 & 0,53 & 0,62 & 0,35 \\
\hline 15 & 0,14 & 0,95 & 2,86 & 0,91 & 2,00 & 0,63 & 0,67 & 0,39 \\
\hline \multicolumn{9}{|c|}{$60-69(n=37)$} \\
\hline \multicolumn{9}{|c|}{ ROC( ${ }^{(\ddagger)}$ área 0,7652 (0,60423-0,92608) } \\
\hline 8,2 & 0,73 & 0,68 & 2,30 & 0,39 & 1,61 & 0,27 & 0,62 & 0,21 \\
\hline 9 & 0,60 & 0,73 & 2,20 & 0,55 & 1,54 & 0,38 & 0,61 & 0,28 \\
\hline 10 & 0,53 & 0,82 & 2,93 & 0,57 & 2,05 & 0,40 & 0,67 & 0,28 \\
\hline 12 & 0,20 & 1,00 & 0,80 & 0,80 & 0,56 & 0,56 & 0,36 & 0,36 \\
\hline 15 & 0,07 & 1,00 & 0,93 & 0,93 & 0,65 & 0,65 & 0,39 & 0,39 \\
\hline \multicolumn{9}{|c|}{$70-79(n=64)$} \\
\hline \multicolumn{9}{|c|}{$\mathrm{ROC}^{(\ddagger)}$ área 0,6225 $(0,47088-0,77408)$} \\
\hline 8,26 & 0,65 & 0,39 & 1,07 & 0,89 & 0,75 & 0,62 & 0,43 & 0,38 \\
\hline 9 & 0,61 & 0,56 & 1,39 & 0,70 & 0,97 & 0,49 & 0,49 & 0,33 \\
\hline 10 & 0,57 & 0,61 & 1,45 & 0,71 & 1,01 & 0,50 & 0,50 & 0,33 \\
\hline 12 & 0,43 & 0,83 & 2,55 & 0,68 & 1,78 & 0,48 & 0,64 & 0,32 \\
\hline 15 & 0,17 & 0,95 & 3,57 & 0,87 & 2,49 & 0,61 & 0,71 & 0,38 \\
\hline \multicolumn{9}{|c|}{$80-89(n=38)$} \\
\hline \multicolumn{9}{|c|}{$\mathrm{ROC}^{(\ddagger)}$ área 0,4931 $(0,30360-0,68255)$} \\
\hline 8,2 & 0,74 & 0,21 & 0,93 & 1,25 & 0,65 & 0,87 & 0,39 & 0,47 \\
\hline 9 & 0,68 & 0,32 & 1,00 & 1,00 & 0,70 & 0,70 & 0,41 & 0,41 \\
\hline 10 & 0,58 & 0,47 & 1,10 & 0,89 & 0,77 & 0,62 & 0,43 & 0,38 \\
\hline 12 & 0,37 & 0,68 & 1,17 & 0,92 & 0,81 & 0,64 & 0,45 & 0,39 \\
\hline 15 & 0,11 & 0,89 & 1,00 & 1,00 & 0,70 & 0,70 & 0,41 & 0,41 \\
\hline
\end{tabular}

(a)Sensibilidad, (b)Especificidad, (c)Razón de verosimilitud positivo, interpretación de la probabilidad de cambio en la condición (> 10, alta; 5-10, moderada; 2-5, baja; 1-2, mínima o nula), (d)Razón de verosimilitud negativo, interpretación de la probabilidad de cambio en la condición (<0,1, alto; 0,1-0,2, moderado; 0,2-0,5, bajo; > 0,5-1, mínimo o nulo), (e)Odd Post-test positivo, ${ }^{(f)}$ Odd Post-test negativo, (g)Probabilidad Post-test positiva, (h) Probabilidad Post-test negativa, (\$)Área bajo la curva (Curva ROC) $(95 \% \mathrm{Cl})$. 


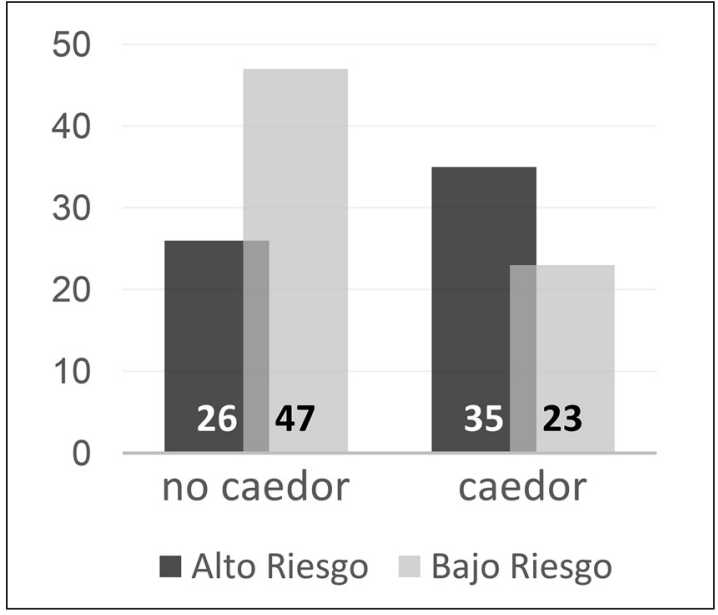

Figura 3. Riesgo de caídas con tiempo de corte en 9 segundos por grupo.

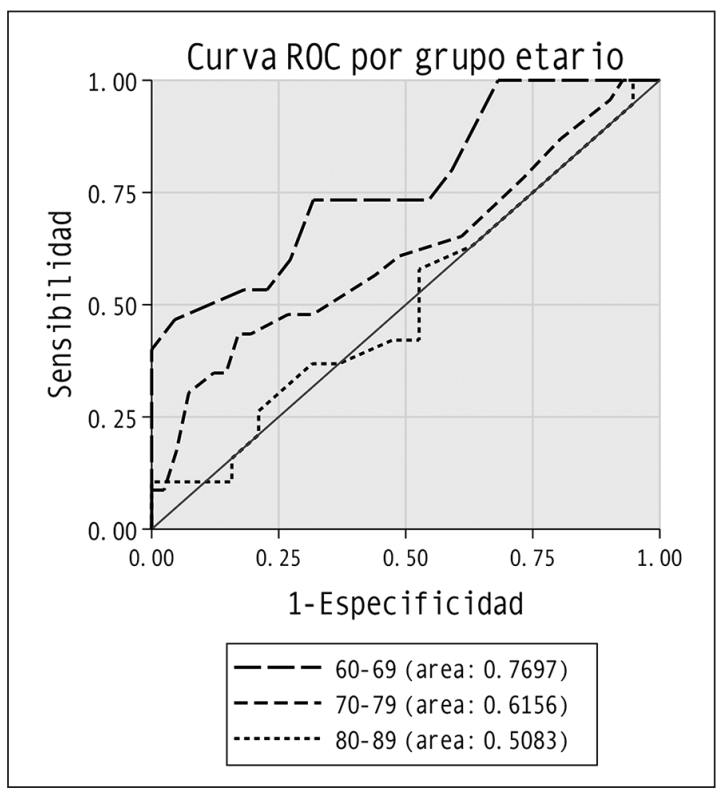

Figura 4. Curvas ROC por grupo etario.

\section{0-69 años}

El análisis de la curva ROC $(S D=0,081$; área $=0,765)$ sugiere que el mejor tiempo de corte es de 8,2 segundos $(S n=0,73 S p=0,68)$. La relación (riesgo de caída / historia de caída), presenta un valor Chi-cuadrado $\chi^{2}=6,15 ; p=0,013$. Esto refleja que el tiempo de corte ( 8,2 segundos) tiene un valor predictivo con una alta $\mathrm{Sn}, \mathrm{Sp}$, y valor asociativo (Figura 5).

\section{0-79 años}

En este grupo, el análisis de la curva ROC $(\mathrm{SD}=0,076$; área $=0,622)$ sugiere que el mejor tiempo de corte es de 9 segundos $(\mathrm{Sn}=0,61$; $\mathrm{Sp}=0,44)$. El valor Chi-cuadrado para el nuevo grupo (fijado en 9 segundos) y este grupo (caedores y no caedores) es $\chi^{2}=0,93 \mathrm{p}=0,332$, reflejando que no existe asociación estadística.

\section{0-89 y 90-99 años}

Para el grupo 80-89 años, el análisis de la curva ROC refleja un área bajo la curva $<0,5$, de manera tal que no existe valor asociativo en este rango.

En el último grupo, solo existieron dos casos, de tal forma que no fue posible un análisis. Su inclusión solamente tuvo fines descriptivos.

\section{Discusión}

El resultado principal de esta investigación es que ambas, Sn y Sp del TUG, pueden aumentar su valor, seleccionando tiempos de corte menores que los aceptados convencionalmente, especialmente en el rango etario 60-69 años. Lo anterior puede sustentarse en dos argumentos: 1) el efecto deletéreo del envejecimiento en este grupo no es tan evidente, contrariamente a los AM caedores dentro del mismo, los cuales conforman $41 \%$ de la muestra en este decenio, y en donde los factores asociados a caídas afectan su rendimiento

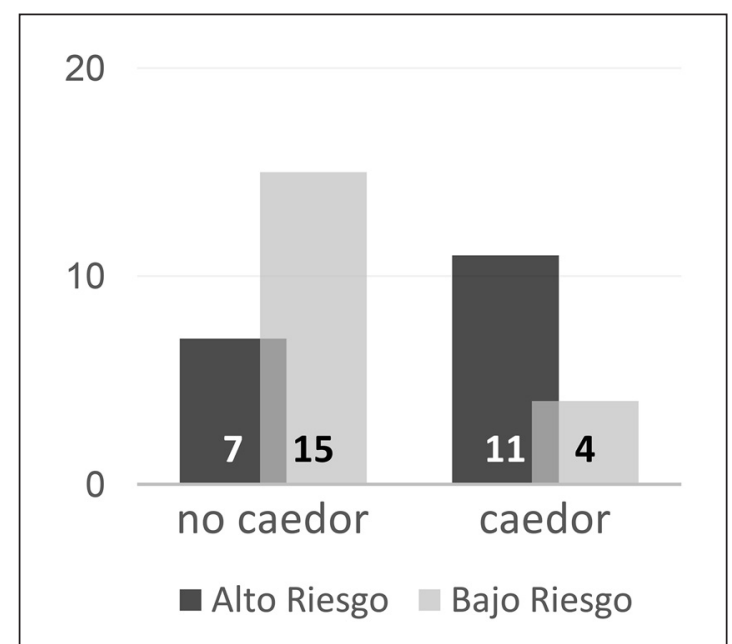

Figura 5. Riesgo de caídas con tiempo de corte en $8,2 \mathrm{~s}$ por historial de caídas en 60-69 años. 
en la prueba. 2) La diferencia en el tiempo de rendimiento de la prueba, y de la velocidad de su ejecución, entre caedores y no caedores, puede ser más patente en este decenio, mejorando de esta forma la capacidad predictiva del TUG. Así mismo, creemos que la aplicación del TUG debe ser complementada con otras pruebas, las cuales deben incluir evaluación cognitiva. Además, el rendimiento en la prueba, globalmente, reflejó que aquellos AM con historias de caídas, tenían peores rendimientos.

Las alteraciones cognitivas incrementan el riesgo de caídas en AM. Las actividades de la vida diaria requieren funciones cognitivas como atención y memoria. Muir-Hunter \& Wittwer ${ }^{15}$ concluyeron que las caídas recurrentes dentro de 12 meses en AM están asociadas a velocidades de marcha más lentas cuando los AM caminan realizando sustracciones de números. Estos hallazgos son similares a los obtenidos en otro estudio, el cual concluye que una tarea dual es útil para predecir riesgo de caídas ya que simula mejor las actividades diarias que desempeñan los $\mathrm{AM}^{15-17}$. Creemos que el valor del TUG reside en su simpleza, y sumándole funciones cognitivas podría mejorar su utilidad sin aumentar su dificultad. La muestra utilizada no presentaba problemas cognitivos (Puntaje Mini-Mental 16,1 + -3,0), y además, no se sumaron tareas cognitivas durante nuestra evaluación.

Aunque el TUG por su diseño, también evalúa fuerza muscular de extremidades inferiores, es insuficiente a la luz de estos argumentos. En efecto, la debilidad de extremidades superiores e inferiores se correlaciona con el riesgo de caídas, y una forma típica de evaluar la fuerza de extremidades inferiores es incorporarse desde una silla, por ejemplo, 5 veces (Test pararse-sentarse), movimiento considerado en la ejecución del TUG ${ }^{13}$. No obstante, el problema del TUG apunta hacia los tiempos de corte utilizados como valores predictivos, o para determinar el nivel de funcionalidad de la población evaluada. Al igual que otros estudios, esta investigación afirma que los tiempos de corte pueden generar confusión ya que muchos de los resultados caen dentro de un estrecho rango de tiempo $(8,9-9,9 \mathrm{~s})^{8}$. En un estudio con AM chilenos, se concluyó que el promedio de rendimiento del TUG de ambos sexos fue de $8,86 \pm 3,58 \mathrm{~s}^{18}$. Sin embargo, los autores no detallan el nivel de funcionalidad de los AM, o si eran caedores o no caedores. Por otro lado, el TUG asociado con otras condiciones basales como género, dolor articular o condiciones como glaucoma y/o cataratas o polifarmacia se asocia con una mejor predicción del riesgo de caídas. El estudio de Ibrahim y cols. ${ }^{19}$, establece tiempos de corte de 11,18 segundos, considerando las variables discutidas anteriormente. Este problema aparentemente reside en que los tiempos de corte dependen de cada población, sin valores únicos. Lo anterior reside en que la distribución de los tiempos del TUG entre caedores y no caedores se solapan más de lo esperado. La complejidad aumenta cuando se considera un valor único de tiempo de corte para todos los AM sin considerar edad. Nosotros observamos que los tiempos de corte deben cambiar entre decenios, observando que el mejor criterio estadístico ( $\mathrm{Sn}$ y $\mathrm{Sp}$ ) se alcanza entre los 60-69 años, en donde se observa una fuerte asociación con el riesgo de caídas, y la clara distinción entre caedores y no caedores, si se utiliza un tiempo de corte de 8,2 segundos.

Mancilla, Valenzuela y Escobar ${ }^{18}$, establecieron un tiempo promedio de $8,86+/$ - 3,58 segundos en 860 AM chilenos sin diferencias entre género, pero con diferencias en edad, lo cual respalda nuestros resultados. Kojima y cols ${ }^{1.0}$ presenta un tiempo de corte de 12,6 segundos para predecir riesgo de caídas, mientras que Ibrahim y cols. ${ }^{19}$ propone un tiempo de corte de 11,18 segundos. A pesar de los tiempos de corte, creemos que el valor predictivo de esta prueba, se incrementa al separar su aplicación por rango etario, o distribuyendola por decenios y complementándola con otras tareas o variables motoras y cognitivas, considerando co-morbilidades de los AM evaluados de acuerdo a lo mencionado anteriormente ${ }^{16-19}$.

La simplicidad del TUG permite su aplicación masiva en AM y puede perfilarse como una herramienta útil para evaluar habilidades motoras más gruesas con todas las variables derivadas de estas, pero teniendo en cuenta que no considera los factores extrínsecos que se conforman como factores detonantes de caídas. Mientras existen otras pruebas que pueden evaluar gestos motores similares a un contexto real en el cual muchas veces ocurren caídas, el TUG es útil como herramienta generalizada para evaluar a grandes poblaciones. Hemos observado que el TUG es una herramienta que puede predecir riesgo de caídas en AM cuando se consideren algunas variables 
como la edad y factores cognitivos. A su vez, la simplicidad de su ejecución, administración y categorización la mantienen como una prueba icónica en el mundo clínico.

Agradecimientos: Agradecemos al Servicio Nacional del Adulto Mayor (SENAMA) por su disponibilidad a colaborar en este proyecto y a los adultos mayores que participaron con entusiasmo.

\section{Referencias}

1. Mathias S, Nayak US, Isaacs B. Balance in elderly patients: the "get-up and go" test. Arch Phys Med Rehabil. 1986; 67 (6): 387-9. http://www.ncbi.nlm.nih.gov/pubmed/3487300.

2. Podsiadlo D, Richardson S. The Timed "Up \& Go": A Test of Basic Functional Mobility for Frail Elderly Persons. J Am Geriatr Soc. 1991; 39 (2): 142-8. doi:10.1111/j.1532-5415.1991.tb01616.x.

3. Dunning K. Timed Up and Go Test. In: Encyclopedia of Clinical Neuropsychology. Cham: Springer International Publishing; 2018: 3466-7. doi:10.1007/978-3-31957111-9_1969.

4. Mathis RA, Taylor JD, Odom BH, Lairamore C. Reliability and Validity of the Patient-Specific Functional Scale in Community-Dwelling Older Adults. J Geriatr Phys Ther. 2019; 42 (3): E67-E72. doi:10.1519/ JPT.0000000000000188.

5. Alghadir A, Anwer S, Brismée JM. The reliability and minimal detectable change of Timed Up and Go test in individuals with grade 1-3 knee osteoarthritis. BMC Musculoskelet Disord. 2015; 16 (1): 1-7. doi: 10.1186/ s12891-015-0637-8.

6. Schoene D, Wu SMS, Mikolaizak AS, et al. Discriminative ability and predictive validity of the timed up and go test in identifying older people who fall: Systematic review and meta-analysis. J Am Geriatr Soc. 2013. doi: 10.1111/jgs.12106.

7. Shumway-Cook A, Brauer S, Woollacott M. Predicting the probability for falls in community-dwelling older adults Using the Timed Up \& Go Test. Phys Ther. 2000; 80 (9): 896-903. doi: 10.1093/ptj/80.9.896.

8. Bohannon RW. Reference Values for the Timed Up and Go Test. J Geriatr Phys Ther. 2006; 29 (2): 64-8. doi: 10.1519/00139143-200608000-00004.

9. Asai T, Oshima K, Fukumoto Y, Yonezawa Y, Matsuo A, Misu S. Association of fall history with the Timed Up and Go test score and the dual task cost: A cross-sectional study among independent community-dwelling older adults. Geriatr Gerontol Int. 2018; 18 (8): 1189-93. doi:10.1111/ggi.13439.

10. Kojima G, Masud T, Kendrick D, et al. Does the timed up and go test predict future falls among British community-dwelling older people? Prospective cohort study nested within a randomised controlled trial. BMC Geriatr. 2015; 15 (1): 1-7. doi:10.1186/s12877-015-0039-7.

11. Barry E, Galvin R, Keogh C, Horgan F, Fahey T. Is the Timed Up and Go test a useful predictor of risk of falls in community dwelling older adults: A systematic review and meta- analysis. BMC Geriatr. 2014; 14 (1): 1-14. doi:10.1186/1471-2318-14-14.

12. Michael YL, Lin JS, Whitlock EP, et al. Interventions to Prevent Falls in Older Adults: An Updated Systematic Review. Evid Synth No 80 AHRQ Publ No 11-05150_ EF-1. 2010; (80).

13. Moreland JD, Richardson JA, Goldsmith CH, Clase CM. Muscle weakness and falls in older adults: A systematic review and meta-analysis. J Am Geriatr Soc. 2004; 52 (7): 1121-9. doi:10.1111/j.1532-5415.2004.52310.x.

14. Nordin E, Lindelöf N, Rosendahl E, Jensen J, Lundin-Olsson L. Prognostic validity of the Timed Upand-Go test, a modified Get-Up-and-Go test, staff's global judgement and fall history in evaluating fall risk in residential care facilities. Age Ageing. 2008; 37 (4): 442-8. doi:10.1093/ageing/afn101.

15. Muir-Hunter SW, Wittwer JE. Dual-task testing to predict falls in community-dwelling older adults: A systematic review. Physiother (United Kingdom). 2016; 102 (1): 29-40. doi: 10.1016/j.physio.2015.04.011.

16. Muhaidat J, Kerr A, Evans JJ, Pilling M, Skelton DA. Validity of simple gait-related dual-task tests in predicting falls in community-dwelling older adults. Arch Phys Med Rehabil. 2014; 95 (1): 58-64. doi: 10.1016/j. apmr.2013.07.027.

17. Cardon-Verbecq C, Loustau M, Guitard E, et al. Predicting falls with the cognitive timed up-and-go dual task in frail older patients. Ann Phys Rehabil Med. 2017; 60 (2): 83-8. doi: 10.1016/j.rehab.2016.07.003.

18. Mancilla SE, Valenzuela HJ, Escobar CM. Rendimiento en las pruebas "Timed Up and Go" y "Estación Unipodal" en adultos mayores chilenos entre 60 y 89 años. Rev Med Chile 2015; 143 (1): 39-46. doi: 10.4067/S003498872015000100005-

19. Ibrahim A, Ajit DKS, Shahar S, Omar MA. Timed up and go test combined with self-rated multifactorial questionnaire on falls risk and sociodemographic factors predicts falls among community-dwelling older adults better than the timed up and go test on its own. J Multidiscip Healthc. 2017; 10: 409-16. doi:10.2147/JMDH. S1425. 